German Business Panel

\title{
Wirkung und Zielgenauigkeit der staatlichen Hilfen für Unternehmen in der Krise
}

\section{Executive Summary}

Bischof | Simons | Voget | Dörrenberg | Rostam-Afschar | Buhlmann | Akari | Arnemann |

Eble | Gharbi | Karlsson

Das German Business Panel ist ein langfristiges Befragungspanel des

DFG-geförderten überregionalen Projektes „Accounting for Transparency“

DFG 


\section{Autoren}

Prof. Dr. Jannis Bischofa, Prof. Dr. Dirk Simonsa, Prof. Dr. Johannes Vogeta, Prof. Dr. Philipp Dörrenberga, Dr. Davud Rostam-Afschara Dr. Florian Buhlmann $n^{b}$, Phares Akaria, Laura Arnemannª, Fabian Eblea, Sarah Gharbia, Christopher Karlssonª

\section{German Business Panel}

Das German Business Panel ist ein langfristiges Befragungspanel des DFG-geförderten

überregionalen Projektes "Accounting for Transparency“"

Wir erheben systematisch und repräsentativ, wie Unternehmen unterschiedlicher Größenordnung Fragen der Unternehmenstransparenz einschätzen. Erhoben wird auch, wie sich die zunehmende Bereitstellung von Informationen sowie die zunehmende Regulierung in den Bereichen Rechnungswesen und Besteuerung auf Entscheidungen in Unternehmen und die Öffentlichkeit auswirken. Als umfassendes, langfristig angelegtes Befragungspanel werden wir Daten in einer bisher nicht dagewesenen

Das German Business Panel ist Teil des Sonderforschungsbereichs. „RR 266 Accounting for Transparency“"

Leitung des German Business Panels:

Principal Investigators:

$$
\text { Research Fellow: }
$$

Projektleitung:

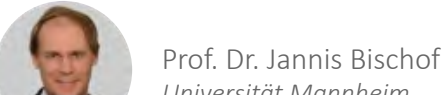

Prof. Dr. Jannis Bischof
jbivchistof @unimnheim
annnheim.de
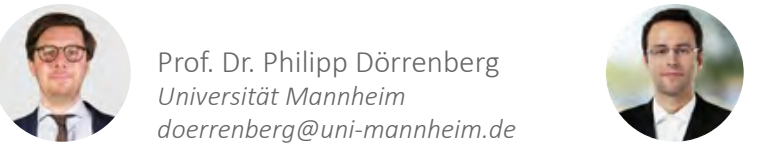

Dr. Davud Rostam-Afschar

Prof. Dr. Dirk Simons

Universität Mannheim
simons@uni-mannheim

Prof. Dr. Johannes Voget

Universität Mannheim
voget@uni-mannheim.de

www.gbpanel.org | gbpinfo@mail.uni-mannheim.de

\section{Inhaltsverzeichnis}

\section{Wichtigste Ergebnisse}

\section{I - Ergebnisse auf Unternehmensebene}

1. Inanspruchnahme staatlicher Leistung und Rettung von Unternehmen

2. Krisenstrategien nach Inanspruchnahme von Staatshilfen

3. Überlebenserwartung nach Betroffenheit im Umsatz

4. Erwartungen in Bezug auf Dauer der Corona Einschränkungen und zweite Welle

\section{II - Ergebnisse auf Branchenebene}

1. Gewinneinbruch nach Branche

2. Zielgenauigkeit der staatlichen Maßnahmen

3. Überlebenswahrscheinlichkeit vs. Betroffenheit

4. Überlebenswahrscheinlichkeit vs. Inanspruchnahme

\section{TRR 266 Accounting for Transparency}

Der TRR 266 Accounting for Transparency ist ein von der Deutschen Forschungsgemeinschaft (DFG) geförderter überregionaler Sonderforschungsbereich.

Unser Team von engagierten Forschenden untersucht, wie Rechnungswesen und Besteuerung die Transparenz von Unternehmen beeinflussen und wie sich Regulierungen und Unternehmenstransparenz auf Wirtschaft und Gesellschaft auswirken. Ziel ist es, eine wirksame Regulierung fur Unternehmenstransparenz und ein transparentes Steuersystem zu entwickeln. In unserer eigenen Forschung gewährleisten wir die Transparenz ebenfalls.

Am TRR 266 sind mehr als 80 Wissenschaftlerinnen und Wissenschaftler beteiligt - von den Hauptstandorten: Universität Paderborn (Sprecherhochschule), Humboldt-Universität zu Berlin und Universität Mannheim, sowie Forscherinnen und Forscher School of Finance \& Management, Goethe-Universität Frankfurt am Main, und WHU - Otto Beisheim School of Management. 


\section{Ziel der Studie}

Die Corona-Krise stellt Unternehmen in Deutschland vor beispiellose Herausforderungen. Das German Business Panel untersucht insbesondere folgende Fragen:

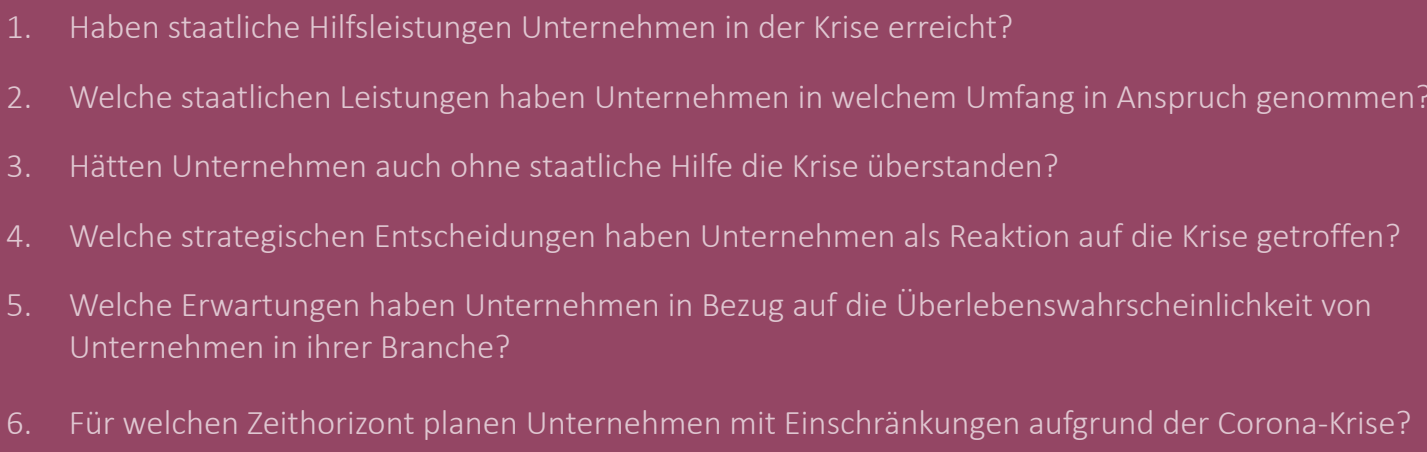

Die Ergebnisse der GBP-Coronabefragung geben Aufschluss darüber, wie zielgerichtet und wie wirksam die staatlichen Hilfsmaßnahmen für Unternehmen waren. Die Ergebnisse sind auf die Grundgesamtheit aller Unternehmen in Deutschland gemäß dem Stand des Unternehmensregisters des Statistischen Bundesamts von 2018 hochgerechnet, soweit nicht anders angegeben.

99

Staatliche Hilfen kommen bei den Unternehmen an, die sie brauchen. Aber sie allein reichen nicht: Viele müssen auf Lohnerhöhungen verzichten, Preise erhöhen oder Arbeitsplätze abbauen. -

\section{Erkenntnisse}

- $2 / 3$ der Unternehmen in Deutschland haben staatliche Hilfen in Anspruch genommen

- Top 3 der staatlichen Hilfen, die in Anspruch genommen sind Corona-Soforthilfe $\quad 49 \%$ Kurzarbeit \& Kurzarbeitergeld $26 \%$ Stundung von Steuerzahlungen $\quad 26 \%$

Die Halfte dieser Unternehmen (48\%) hatte die Krise nach eigener Einschátzung ohne staatliche Hilfe nich überstanden.

- Folgende Maßnahmen ergreifen Unternehmen aufgrund der Krise am häufigsten:

Verminderung von zukünftigen Lohnsteigerungen

Preiserhöhungen

Verminderung einbehaltener Gewinn

Verminderung von Ausschüttungen an Anteilseigner Abbau von Arbeitsplätzen

$32 \%$
$25 \%$
$21 \%$
$18 \%$
$13 \%$

- Maßnahmen, die ergriffen werden, um die Krise kurffristig (0-12 Monate) zu bewältigen:

$\begin{array}{lcc}\begin{array}{l}\text { Geplante Maßnahmen } \\ \text { Verminderung von zukünftigen Lohnsteigerungen } \\ \text { oder Bonuszahlungen }\end{array} & \begin{array}{c}\text { mit } \\ \text { Staatshilfe }\end{array} & \begin{array}{c}\text { ohne } \\ \text { Staatshilfe }\end{array} \\ \text { Abbau von Arbeitsplätzen } & 39 \% & 18 \% \\ \text { Reduktion von Ausschüttungen an Anteilseigner } & 20 \% & 17 \% \\ \text { Verminderung einbehaltener Gewinne } & 23 \% & 12 \% \\ \text { Erhöhung von Preisen } & 27 \% & 16 \% \\ \text { Outsourcing Freelance } & 11 \% & 5 \% \\ \text { Outsourcing Unternehmensteile } & 3 \% & 1 \% \\ \text { Reduktion F\&E } & 7 \% & 3 \% \\ \text { Besteuerung Körperschaftsteuer } & 3 \% & 1 \% \\ \text { Sonstige } & 7 \% & 5 \%\end{array}$

- $\quad \mathbf{5 9}$ \% der Unternehmen erfuhren einen Umsatzrückgang von mindestens $\mathbf{1 0} \%$. Bei $43 \%$ der Unternehmen betrug der Umsatzeinbruch sogar mindestens $30 \%$

- $\mathbf{8 0}$ \% der Unternehmen erwarten eine zweite Welle. Bei der Dauer der Einschränkungen sind die Meinungen geteilt: Etwa die Hälfte der Unternehmen gehen davon aus, dass die Einschränkungen durch die Corona-Krise noch länger als ein Jahr dauern werden.

- Die Reisebranche, Gastronomie und Getränkehersteller sowie Dienstleistungen des Sports, der Unterhaltung sowie der Erholung und kreative, künstlerische und unterhaltende Tätigkeiten haben den stärksten

- Branchen, deren Gewinn stärker durch die Krise betroffen ist, nehmen Staatshilfen eher in Anspruch.
Fazit

Insgesamt haben zwei Drittel der Unternehmen in Deutschland staatliche Hilfen in Anspruch genommen. Damit konnte eine erste Welle an Unternehmensinsolvenzen abgewendet werden. Allerdings erwarten vier von fünf Unternehmen eine zweite Welle und 50 \% rechnen damit, dass sich die Einschränkungen der Krise noch mehr als ein weiteres Jahr negativ auf das wirtschaftliche Leben in Deutschland auswirken werden. Wenn es dazu kommt, könnten weitere Staatshilfen nötig werden. 
Auffällig ist der geringe Anteil (8\%) an Arbeitsplatzabbau von Unternehmen, die bisher ohne Staatshilfen auskamen. Diese scheinen an anderen Stellen wie am zukünftigen Lohnzuwachs (18 \%) zu kürzen und Preise zu erhöhen (16\%), um Arbeitsplätze erhalten zu können.

$\begin{array}{lcc}\text { Geplante Maßnahmen } & \begin{array}{c}\text { mit } \\ \text { Staatshilfe }\end{array} & \begin{array}{c}\text { ohne } \\ \text { Staatshilfe }\end{array} \\ \begin{array}{l}\text { Verminderung von zukünftigen Lohnsteigerungen } \\ \text { oder Bonuszahlungen }\end{array} & 39 \% & 19 \% \\ \text { Abbau von Arbeitsplätzen } & 17 \% & 6 \% \\ \text { Reduktion von Ausschüttungen an Anteilseigner } & 20 \% & 13 \% \\ \text { Verminderung einbehaltener Gewinne } & 24 \% & 14 \% \\ \text { Erhöhung von Preisen } & 29 \% & 19 \% \\ \text { Outsourcing Freelance } & 12 \% & 5 \% \\ \text { Outsourcing Unternehmensteile } & 3 \% & 0 \% \\ \text { Reduktion F\&E } & 8 \% & 2 \% \\ \text { Besteuerung Körperschaftsteuer } & 2 \% & 1 \% \\ \text { Sonstige } & 7 \% & 6 \%\end{array}$

\section{3. Überlebenserwartung nach Betroffenheit im Umsatz}

In der nächsten Abbildung stellen wir den Zusammenhang zwischen Überlebenserwartungen und Betroffenheit durch die Corona-Krise dar. Dieser Zusammenhang basiert auf Variablen, die Antworten auf die Fragen quantifizieren:

1. In welchem Ausmaß wurde Ihr Umsatz durch die Corona-Krise beeinflusst? Bitte geben Sie an um wie viel Prozent sich die Kennzahlen aktuell im Vergleich zum 31.01 .2020 verändert haben. (horizontale Achse)

2. Was schätzen Sie: Wie viel Prozent der Unternehmen Ihrer Branche werden die Corona-Krise bis zum 31.12.2020 überstehen? (vertikale Achse)

Es zeigt sich, dass mit stärkerem Umsatzrückgang eines Unternehmens erwartet wird, dass ein niedrigerer Anteil an Unternehmen die Corona-Krise bis zum 31.12. überstehen. Allerdings stagniert der Effekt ab einem Umsatzeinbruch von ca. -10\%. Unternehmen mit besonders hohen Umsatzzuwächsen sind tendenziell wieder etwas pessimistischer. Dies könnte daran liegen, dass sie diese Umsatzsteigerungen als nicht nachhaltig betrachten. Jeder Punkt in der Abbildung repräsentiert $1 \%$ der Unternehmen gruppiert nach der Betroffenheit (d.h. dass der Punkt, der am weitesten links ist, den Prozentsatz der Unternehmen repräsentiert, die am stärksten betroffen sind). Es wird die Medianantwort gezeigt. In der Gruppe der am stärksten betroffenen Unternehmen denken 50 \%, dass etwa 60 \% der Unternehmen, hrer Branche die Krise bis zum 31.12.2020 überstehen.

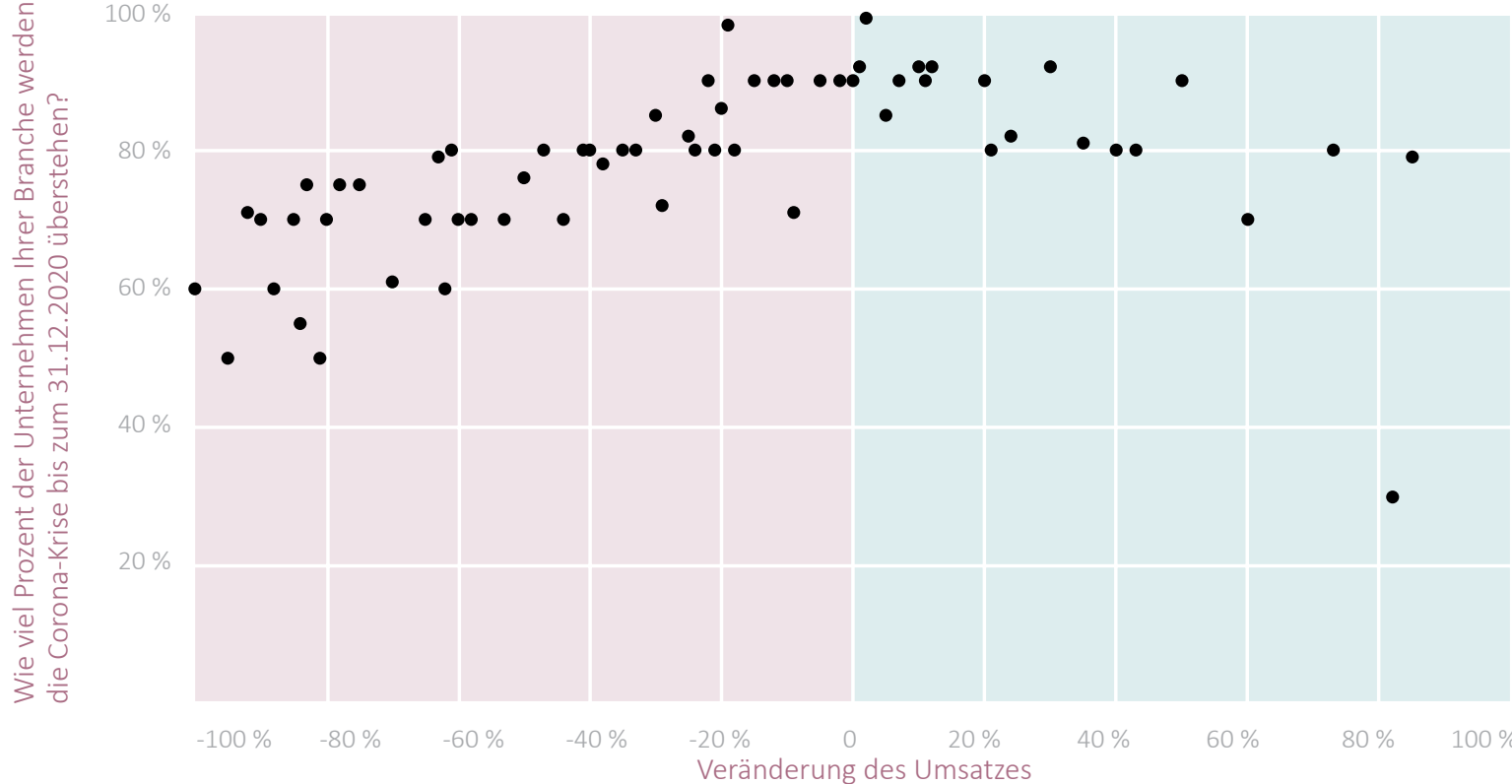

Wie viele Unternehmen hatten einen Umsatzrückgang von mindestens $10 \%, 20 \%$ und $30 \%$ ?

59 \% der Unternehmen hatten einen Umsatzrückgang von mindestens $10 \%, 54$ \% einen Umsatzrückgang von mindestens $20 \%$ und $44 \%$ der Unternehmen einen Umsatzrückgang von mindesten $30 \%$.

\section{Erwartungen in Bezug auf Dauer der Corona-Einschränkungen und zweite Welle}

Frage: Was schätzen Sie: An welchem Datum wird das öffentliche Leben in Deutschland aufgrund der Corona-Krise nicht mehr eingeschränkt sein?

Die folgende Abbildung zeigt die kumulative Verteilung der Antworten. Ungefähr 50 \% der Unternehmen gehen davon aus, dass es länger als ein Jahr dauern wird, bis die Einschränkungen enden.

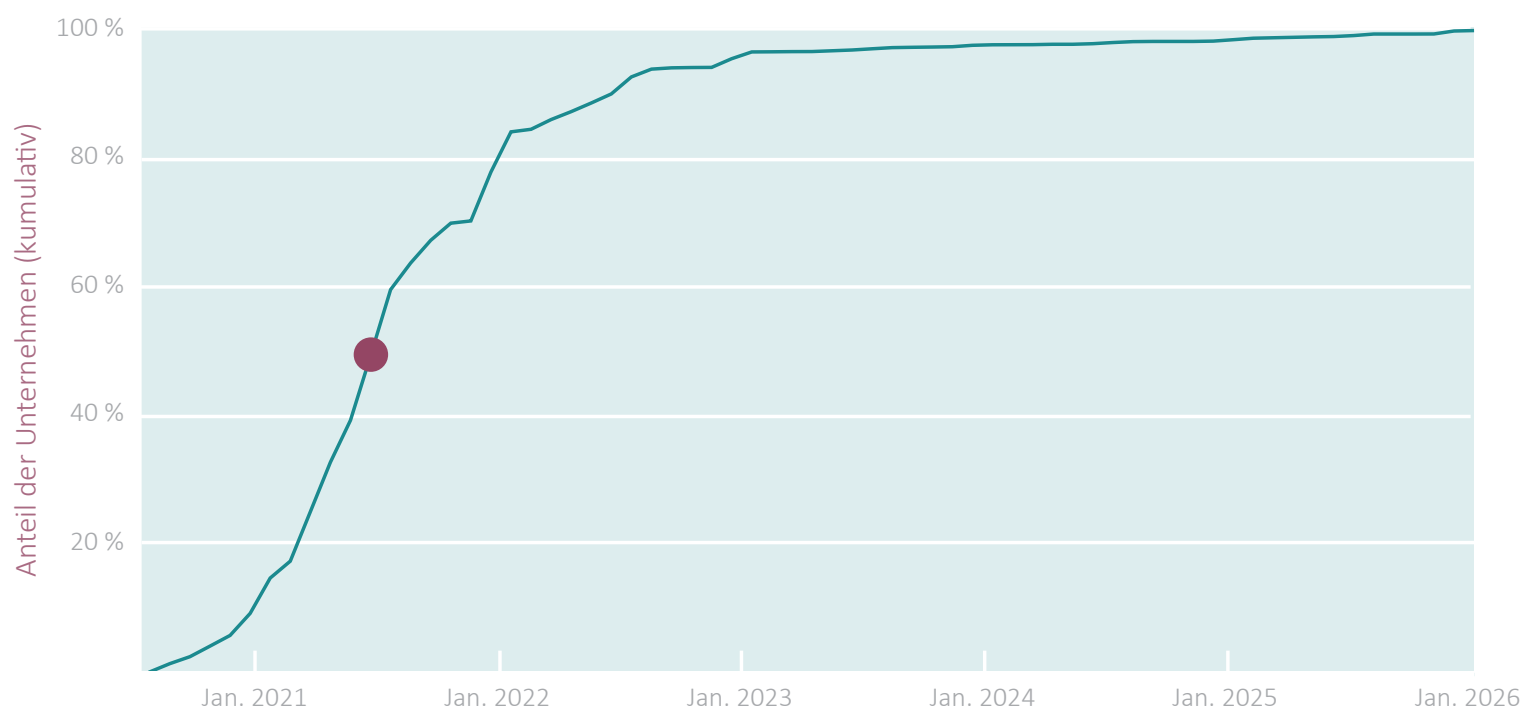


Die folgende Abbildung differenziert die Antworten zur Betroffenheit in Prozent des Umsatzes nach 38 Regierungsbezirken. Dunklere Farben stehen für einen stärkeren Rückgang des Umsatzes. In jedem Regierungsbezirk wird der ungewichtete Median geplottet. Mit der Ausnahme des Regierungsbezirks

Saarland, für den keine Beobachtungen vorlagen, sind in allen Regierungsbezirken mindestens 35 Beobachtungen vorhanden.
Umsatzveränderung

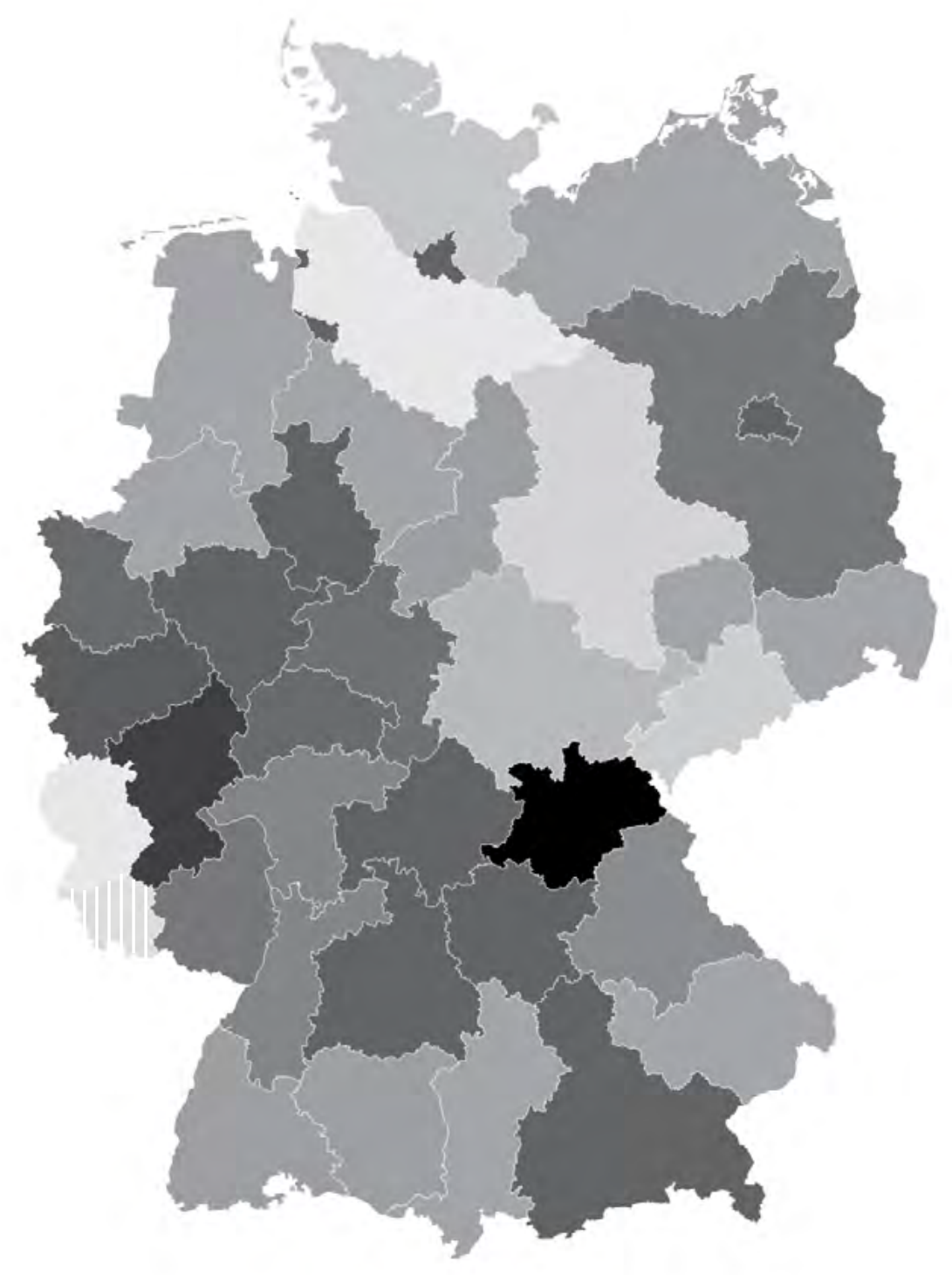

Gewinnveränderung

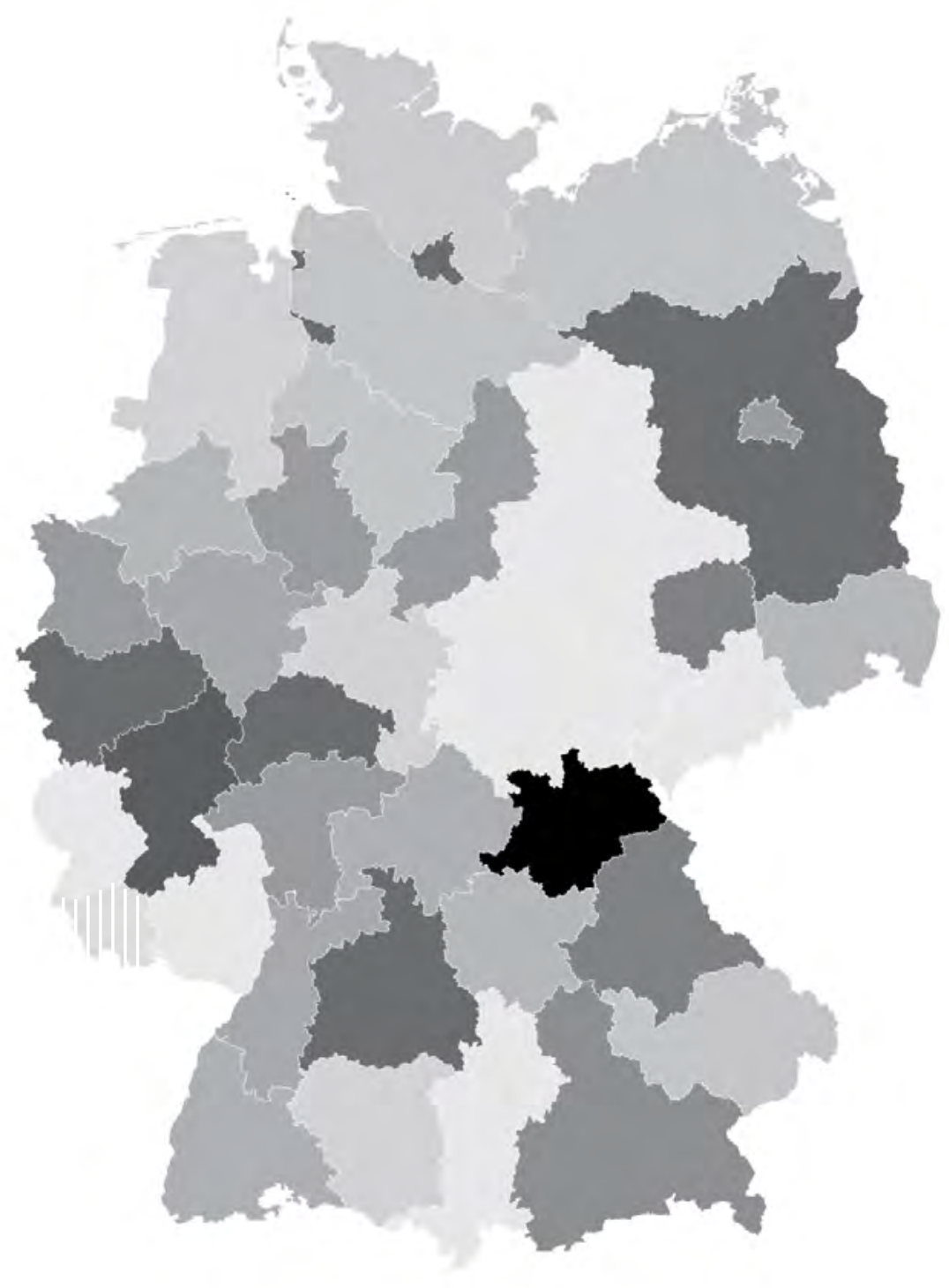

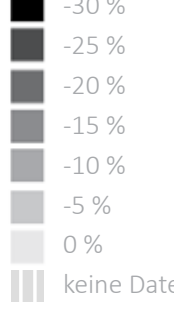

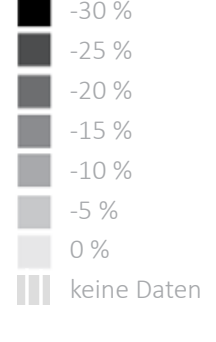


Frage: Erwarten Sie, dass es vor dem Ende der Krise noch zu einer zweiten Welle an Infektionen kommt?

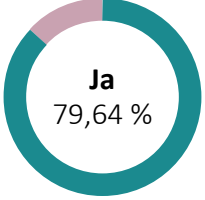

Falls Ja: Was schätzen Sie: An welchem Datum wird die zweite Welle beginnen?

Diese Frage wurde von Unternehmen beantwortet, die die vorherige Frage mit "Ja" beantwortet haben.

Ein interessanter Fakt ist, dass 99 \% der Unternehmen, die mit einer zweiten Welle rechnen, davon ausgehen, dass diese vor dem 1. Januar 2021 kommen wird. Der Median ist der 30.09.2020.

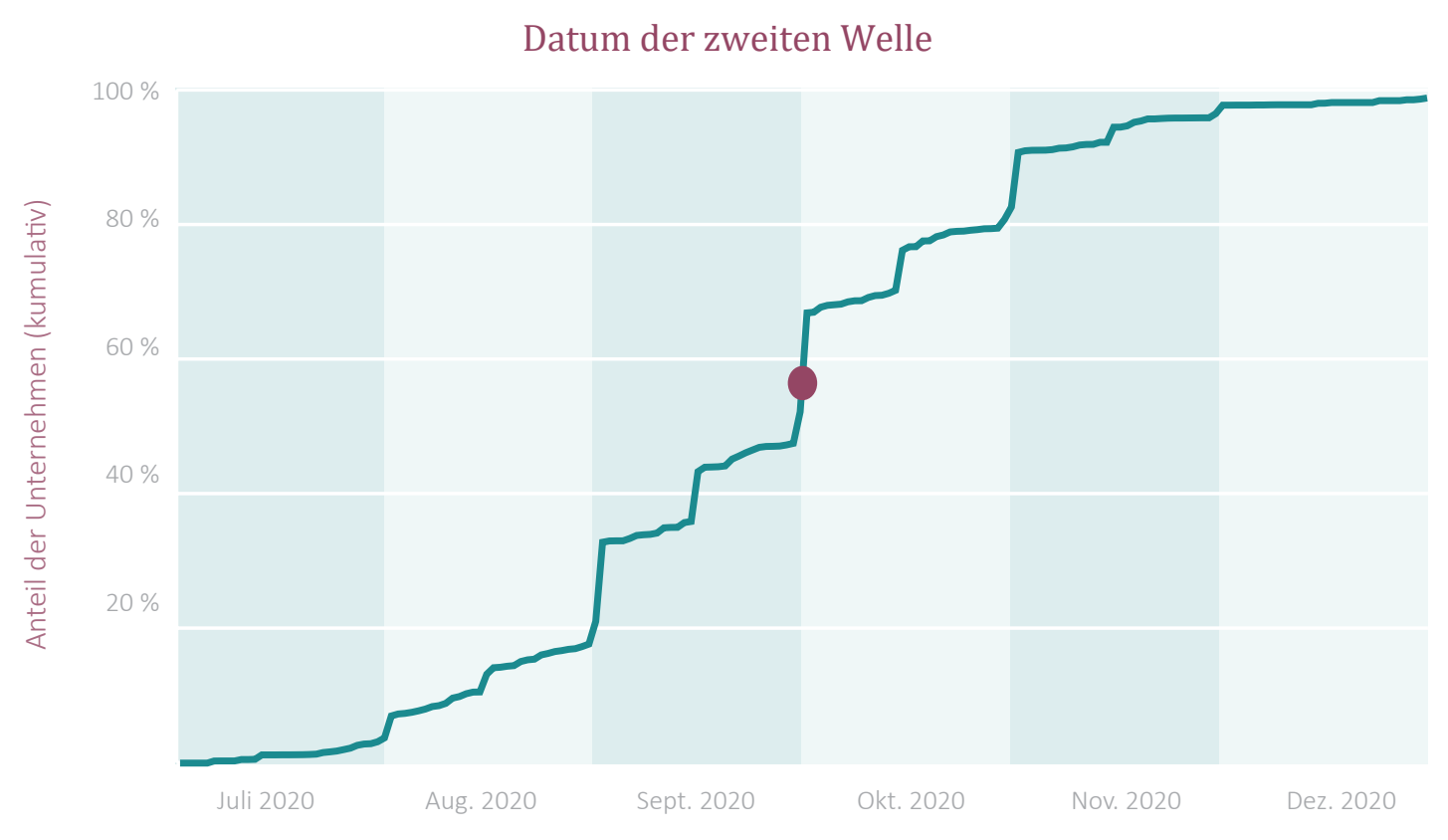

\section{II - Ergebnisse auf Branchenebene}

In den folgenden Abbildungen aggregieren wir die Unternehmen auf Branchenebene. Wir nutzen dazu die Klassifikation der Wirtschaftszweige des Statistischen Bundesamts (WZ2008) auf Zweistellerebene. Unsere Ergebnisse basieren auf 73

Branchen. Wirtschaftszweige mit weniger als 10 Beobachtungen werden aufgrund der erhöhten statistischen Unsicherheit nicht berücksichtigt. Alle Ergebnisse sind auf die Grundgesamtheit aller Unternehmen in Deutschland gemäß dem

Unternehmensregisterstand von 2018 des Statistischen Bundesamtes hochgerechnet soweit nicht anders angegeben.

\section{Gewinneinbruch nach Branche}

Welche Branchen sind am stärksten betroffen? Nach Gewinneinbruch im Vergleich zum 31.01.:

\section{Branche}

Gewinnrückgang
durch Corona

Reisebüros, Reiseveranstalter und Erbringung sonstiger
Reservierungsdienstleistungen

Spiel-, Wett- und Lotteriewesen

$$
-63,6 \%
$$

$-61,8 \%$

Getränkeherstellung $\quad-50,0 \%$

Beherbergung $\quad-49,9 \%$

Gastronomie $\quad-48,8 \%$

Vermietung von beweglichen Sachen $\quad-48.5 \%$

Welche Branchen sind am wenigsten betroffen? Nach Gewinnveränderung im Vergleich zum 31.01.

Branche Gewinnruckgang
durch Corona

\section{Abwasserentsorgung}

$5,6 \%$

Heime (ohne Erholungs- und Ferienheime)

Gebäudebetreuung

Sammlung, Behandlung und Beseitigung von Abfällen

Tiefbau 


\section{Zielgenauigkeit der staatlichen Maßnahmen}

Die folgende Abbildung zeigt, wie stark der Zusammenhang zwischen der Quote der Inanspruchnahme von Staatshilfen innerhalb einer Branche und der durch Corona bedingten Veränderung des Gewinns ist. Jeder Punkt repräsentiert eine Branche und gibt für diese an, wie hoch die durchschnittliche Inanspruchnahme und wie hoch der durchschnittliche Gewinneinbruch war. Es zeigt sich zum einen, dass sich die Quote der Inanspruchnahme nach Branchen stark unterscheidet. Zum anderen zeigt sich, dass die Branchen, die stärker betroffen sind, auch eher Staatshilfen in Anspruch nehmen. Die Grafik liefert einen wichtigen Hinweis darauf, wie zielgenau die Staatshilfen gewirkt haben. In der am stärksten betroffenen Branche (Reisebüros, Reiseveranstalter und Erbringung sonstiger Reservierungsdienstleistungen) lag die Quote der Inanspruchnahme bei fast $100 \%$. In den am wenigsten betroffenen Branchen liegt sie bei nur ca. $30-60 \%$.

\section{Korrelation Inanspruchnahme Staatshilfen und Gewinneinbruch}

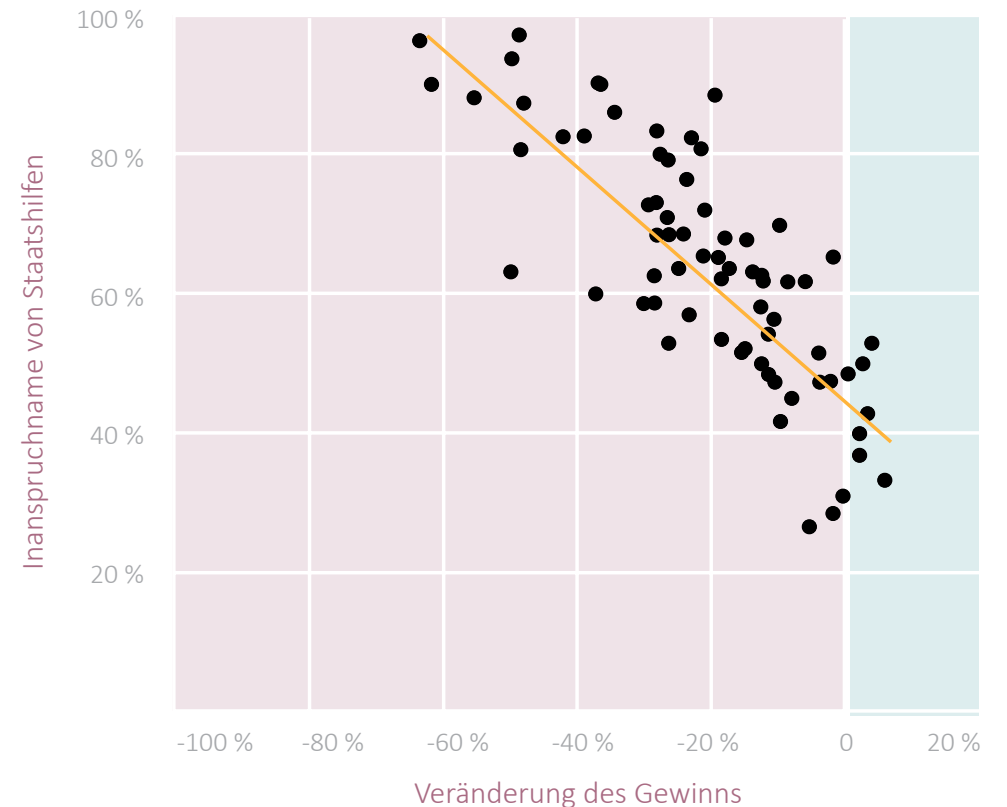

\section{3. Überlebenswahrscheinlichkeit vs. Betroffenheit}

Die nächste Abbildung zeigt, dass der erwartete Anteil der Unternehmen einer Branche, die bis zum 31.12.2020 überleben, steigt, wenn die Branche weniger stark getroffen wurde.

Korrelation Überlebenserwartung zum 31.12 und Gewinn(einbruch)

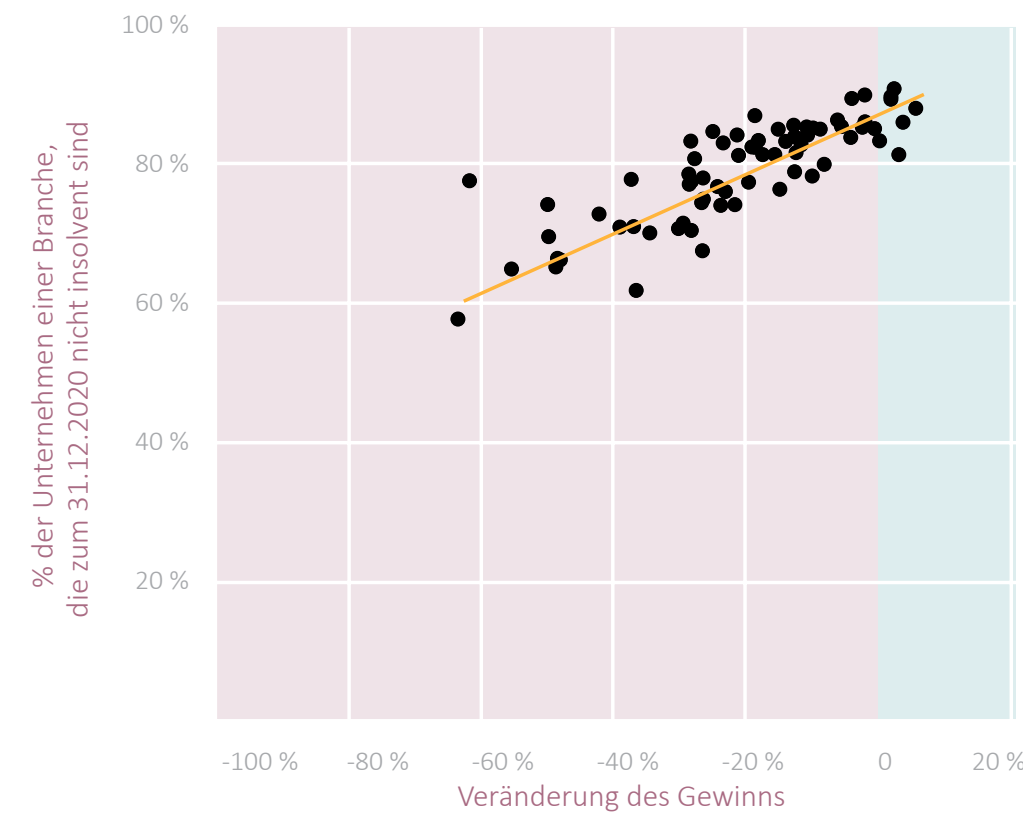

\section{4. Überlebenswahrscheinlichkeit vs. Inanspruchnahme}

Die Abbildung unten zeigt, dass der Zusammenhang zwischen der Quote der Inanspruchnahme und dem erwarteten Anteil der Unternehmen, die am 31.12.2020 noch existieren, negativ ist. Die Grafik sollte allerdings nicht unbedingt kausal interpretiert werden, da stärker betroffene Unternehmen auch eine höhere Wahrscheinlichkeit haben, Staatshilfen in Anspruch zu nehmen. Regressionsergebnisse zeigen, dass sich die Korrelation abschwächt, wenn die Betroffenheit der Branche berücksichtigt wird, sie bleibt aber signifikant. Dies liefert Evidenz dafür, dass mittelfristig weitere Maßnahmen erforderlich sein könnten, dass die Staatshilfen aber kurzfristig gewirkt haben (vgl. die Abbildung „Hätten Sie die Krise ohne staatliche Hilfe überstanden").

Korrelation Überlebenserwartung zum 31.12. und Inanspruchname von Staatshilfen

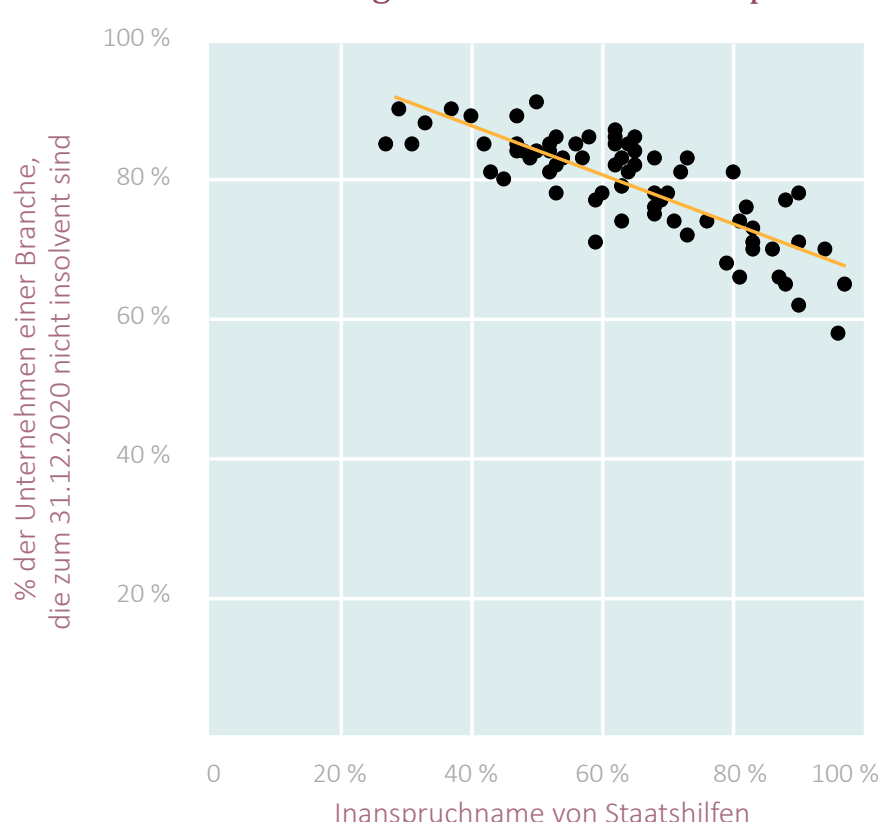




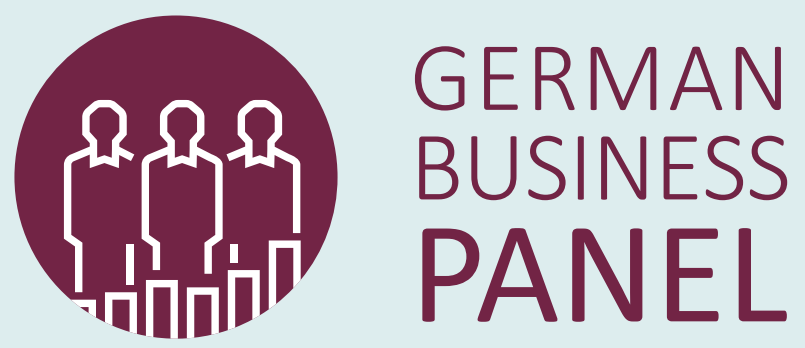

Das German Business Panel ist ein langfristiges Befragungspanel des DFG-geförderten überregionalen Projektes „Accounting for Transparency“ TRANSPARENCY

www.accounting-for-transparency.de www.gbpanel.org

\section{7) $\begin{aligned} & \text { Deutsche } \\ & \text { Forschungsgemeinschaft }\end{aligned}$}

